\title{
Influenza A/H1N1 MF59 adjuvanted vaccine in pregnant women and adverse perinatal outcomes: multicentre study
}

This Research paper by F Rubinstein and colleagues (BMJ 2013;346:f393, doi:10.1136/bmj.f393), contains an error in table 3 . The percentages for perinatal mortality should have read 0.7 for vaccinated women and 1.1 for non-vaccinated women (not for vaccinated women and 1.1 for non-vaccinated
"7.4" and "11.0," which were the rates per 1000). 